\title{
Indirect Coupling Method of Chip Impedance Matched Dipole Antenna for UHF RFID Tag
}

\author{
J. Gandhimohan \\ M.Tech. (Electronics) \\ Department of Electronics Engineering \\ Pondicherry University, Puducherry, India
}

\author{
T. Shanmuganantham \\ Assistant Professor \\ Department of Electronics Engineering \\ Pondicherry University, Puducherry, India
}

\begin{abstract}
Radio Frequency Identification is the wireless technology that uses radio waves to automatically identify items within certain proximity. This process involves tagging items with a transmitter which will send bursts of information. Passive RFID tag consists of a microchip connected with an antenna. The passive tag uses the electromagnetic pulse from readers as a power source to transmit its identifier. So proper impedance matching between the antenna and the chip is very important in passive RFID tag design. The RFID system performance characteristics and the range of a tag depend on the impedance matching. A load impedance of RFID microchip is a nonlinear whose complex part varies with the frequency and the input power [2]. This paper presents simulated results of a UHF RFID dipole antenna and inductively coupled with a small rectangular loop. Such a design provides controlled values of inductive reactance that is required for obtaining good impedance match of the antenna to microchip. The proposed dipole antenna is simple and robust in design, which enables low-cost while realization.
\end{abstract}

\section{General Terms}

Return-loss, 3D Radiation Pattern, 2D Azimuth and Elevation Radiation Pattern, S-Parameters.

\section{Keywords}

Radio Frequency Identification (RFID); Microchip; Impedance; Ultra High Frequency (UHF); Passive.

\section{INTRODUCTION}

In recent years automatic identification procedures have become very popular in many service industries, purchasing and distribution logistics, manufacturing companies and material flow systems. Automatic identification procedures exist to provide information about people, animals, goods and products in transit. RFID is an automatic contactless data collection technology. The idea of early RFID system was invented by Scottish physicist Sir Robert Alexander WatsonWatt in 1935 [10]. RFID systems consist of two components,

* Reader

* Transponder (or Tag).

There are two main classes of RFID systems, depending on characteristics of the transponder,

* Active

* Passive

Passive RFID systems are more frequent in use. Passive RFID tags utilize an induced antenna coil voltage for operation. This induced $\mathrm{AC}$ voltage is rectified to provide a voltage source for the device. As the DC voltage reaches a certain level, the device starts operating. By providing an energizing RF signal, a reader can communicate with a remotely located device that has no external power source such as a battery. Since the energizing and communication between the reader and tag is accomplished through antenna, it is important that the device must be equipped with a proper antenna circuit for successful RFID applications. Active transponders incorporate a battery which supplies the power for the operation.

\section{DESIGN GOALS}

Proper impedance match between the antenna and the chip is paramount importance in RFID. Since new IC design and manufacturing is a big and costly venture, RFID tag antennas are designed for a specific microchip available in the market. Adding an external matching network with lumped elements is usually prohibitive in RFID tags due to cost and issues [8]. To overcome this situation, antenna can be directly matched to the chip which has complex impedance varying with the frequency. So, the antenna should be conjugate match to the chip i.e. $\mathrm{Za}=\mathrm{Zc},(\mathrm{Za}=(\mathrm{Ra}+\mathrm{jXa})$ Antenna Impedance and $\mathrm{Zc}=(\mathrm{Rc}+\mathrm{jXc})$ Microchip or Source Impedance $)$, which allow the tag antenna to receive the maximum power from the reader's antenna and sent out the strongest signal [2]. Therefore, in designing the tag antenna, we need to tune the dimensions of the antenna to achieve $\mathrm{Za}=\mathrm{Zc}$ at frequency range of interests to achieve conjugate match. Secondly, there is only a fraction of the power delivered to antenna will be radiated out. So, we also need to achieve highest radiation efficiency. The resonant frequency of the tag is at where $\mathrm{Xa}=-\mathrm{Xc}$ and Ra close to Rc, which means the tag and antenna are conjugate matched.

\section{DESIGN METHODOLOGY}

We start by considering the imaginary part to be high, the self-resonant frequency of the coupling source antenna should approach the operating frequency fr of the composite antenna. In the present design, parameters of the coupling source are used to control its inductive reactance. The distance between the coupling source and radiating elements are controls the real part of the antenna impedance. The stub elements presents over the radiator dipole element are used to tune the resonance frequency of the composite antenna and to improve the reflection coefficients.

\subsection{Performance criteria}

The most important performance characteristic of a RFID tag is the read range. Read range is the maximum distance at which RFID reader can detect the backscattered signal from 
the tag. We can use Friis free - space equation to calculate the tag's read range ' $r$ ' as

$$
\mathrm{r}=\frac{\lambda}{4 \pi} \sqrt{\frac{P_{\mathrm{r}} \mathrm{G}_{\mathrm{r}} \mathrm{G}_{\mathrm{Y}} \mathrm{W}}{\mathrm{P}_{\mathrm{th}}}}
$$

Where $\lambda$ is the wavelength, $\mathrm{Pt}$ is the power transmitted by the reader, Gt is the gain of the transmitting antenna, $\mathrm{Gr}$ is the gain of the receiving tag antenna, $\mathrm{Pt}$ is the minimum threshold power necessary for the RFID tag chip and $\gamma$ is the power transmission coefficient [9]. The power transmission coefficient is given as

$$
\mathbb{W}=\frac{4 \operatorname{RcRa}}{\left.\|[\mathrm{Z} a+\mathrm{Zc}]^{2}\right]^{2}} \quad 0 \leq \mathbb{W} \leq 1
$$

where $\mathrm{Za}=\mathrm{Ra}+\mathrm{jXa}$ is the antenna impedance and $\mathrm{Zc}=$ $\mathrm{Rc}+\mathrm{jXc}$ is the chip impedance. The better matching between the antenna and chip impedances will achieve a high power transmission coefficient (closer to 1) [8]. A good RFID tag should have a high transmission coefficient above 0.7 [8]. In order to achieve high power transmission coefficient, the antenna and chip impedances should be conjugated to each other, i.e. $\mathrm{Za}=\mathrm{Zc}$. So, if the antenna and chip is perfectly conjugated match, it will have a power transmission coefficient of 1 [8]. From Friis free - space equation, the tag read range is determined by the product $\mathrm{Pt}$ and $\mathrm{Gt}$ of the reader (transmitter EIRP), tag antenna gain $\mathrm{Gr}$ and transmission coefficient $\gamma$ (which is determined by the antenna and chip impedance). The frequency of the peak range is referred as tag resonance, which happens at the frequency of the best impedance match between chip and antenna.

\subsection{Dipole Antenna for $900 \mathrm{MHz}$}

A RFID antenna designed for $900 \mathrm{MHz}$ is about one half wavelengths long. A half wavelength antenna is known as a dipole. A dipole is a very good radiator. RFID tags using dipoles at $900 \mathrm{MHz}$ have a range of up to 10 meters. This works well for large pallets of product. Unfortunately, there is not yet a worldwide frequency allocation for RFID at 900 MHz. Europe and US have different allocations fairly close to $900 \mathrm{MHz}$. Japan is considering an allocation near $960 \mathrm{MHz}$. The design example for this presentation assumes $900 \mathrm{MHz}$. Once the RF output power of the reader and its antenna gain are fixed, the performance of the RFID tag is mainly determined by the conjugate matching condition between the RFID chip and the antenna. Therefore, a successful antenna design must achieve a good conjugate impedance match condition between the chip and the antenna. The indirect coupling approach is helpful for solving the impedance matching problem, which entails the conjugate matching of a high quality factor antenna, the resistance of which is low but the reactance is high. The parameters of both the coupling source and the radiating body can be used to control the input impedance characteristics of the composite.

\subsection{Antenna configuration}

Fig. 1 shows the configuration of the proposed indirect coupling method for the design of the RFID tag. The antenna comprises a radiating body and the coupling source, which is a small dipole, is treated as a dipole antenna of arbitrary length. The characteristics of the input impedance of the proposed antenna are shown in Fig. 7. Thus, the desired input impedance of the antenna can be realized not only by tuning the dimensions of the radiating body but also by adjusting the dimensions of the coupling source.
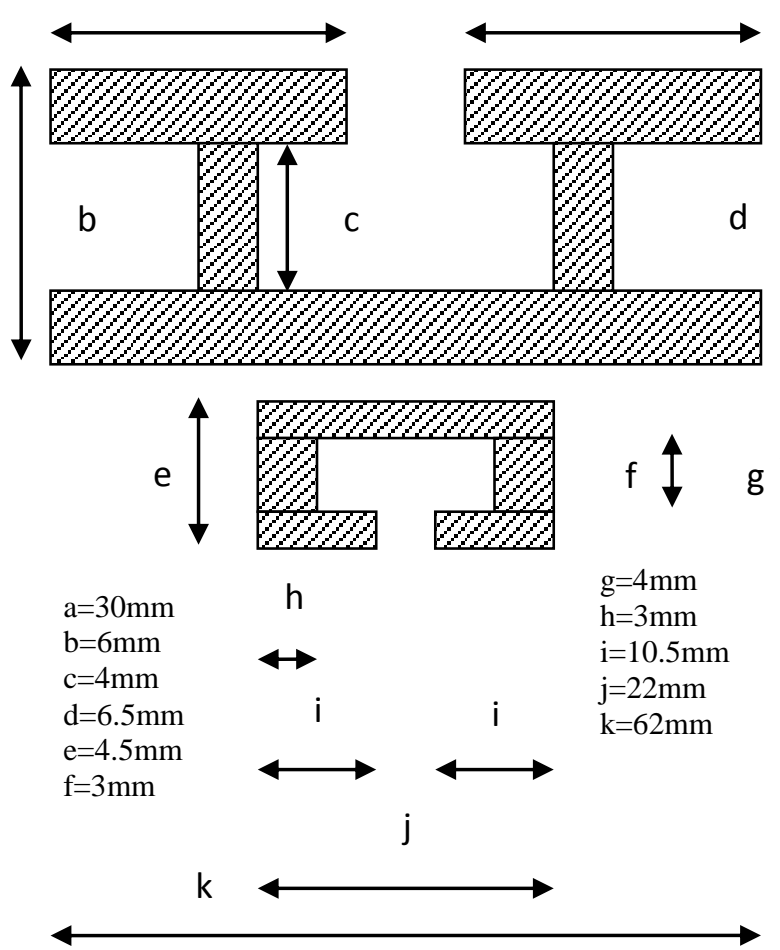

Fig.1 Proposed Antenna Configuration a

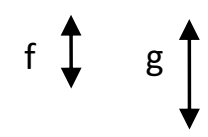
$\mathrm{i}=10.5 \mathrm{~mm}$ $\mathrm{j}=22 \mathrm{~mm}$ $\mathrm{k}=62 \mathrm{~mm}$

\subsection{Design Example}

The proposed RFID tag antenna design is shown in Fig. 1. The antenna is designed for the RFID chip encased in a strap package, with an input impedance of (50-j120 $\square)$ at $900 \mathrm{MHz}$. The configuration is printed on an FR4 substrate, with thickness of $0.4 \mathrm{~mm}$, relative permittivity of 4.4 and a dielectric loss tangent of 0.002 . The geometry is simulated using Zeland IE3D software.

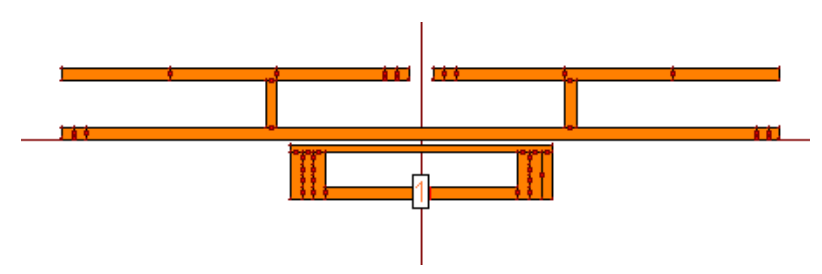

Fig.2 Proposed Antenna Design in IE3D

\section{SIMULATION RESULTS}

The design goal is to achieve Ra and Xa as close to Rc and $\mathrm{Xc}$ as possible respectively. From the result, we can see that at $900 \mathrm{MHz}, \mathrm{Xa}$ is 125 which are very close to $-\mathrm{Xc}(120), \mathrm{Ra}$ is 60 , when compare with Rc (50), the difference is small. The smith chart and the Input Impedance Characteristic of the simulated results shows the input impedance of the proposed antenna. The conjugate matching factor shows the closeness of the proposed antenna Input Impedance with required value of Input Impedance. Omnidirectional radiation pattern has been observed from the $2 \mathrm{D}$ and $3 \mathrm{D}$ radiation pattern graph of the proposed antenna.

The following figures show the various simulated results of the proposed antenna. 


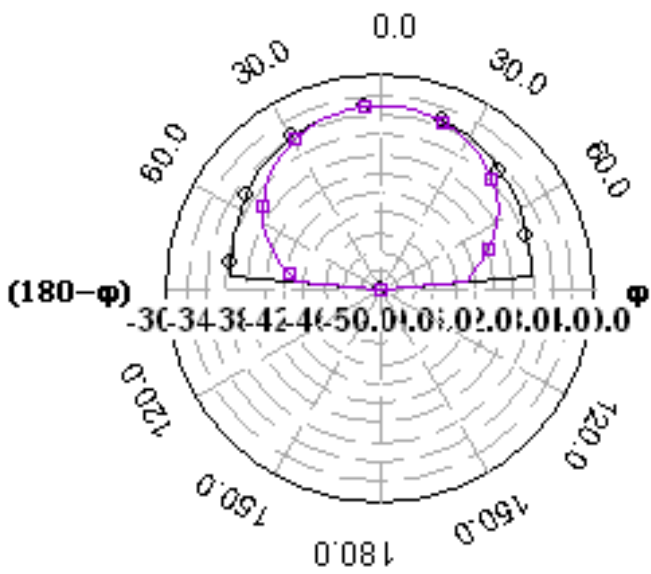

Elevation Pattern Gain Display (dBi)

Fig.3 2D Elevation Pattern
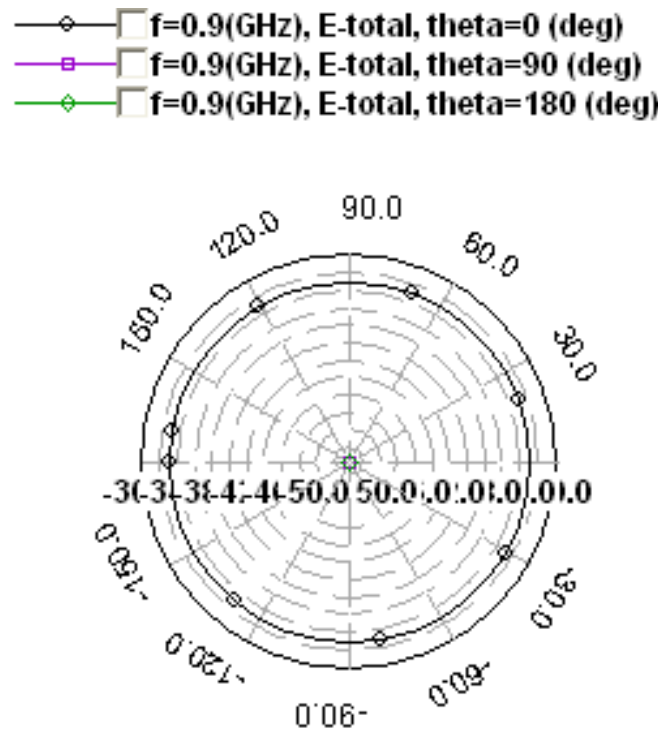

Azimuth Pattern Gain Display (dBi)

Fig.4 2D Azimuth Pattern

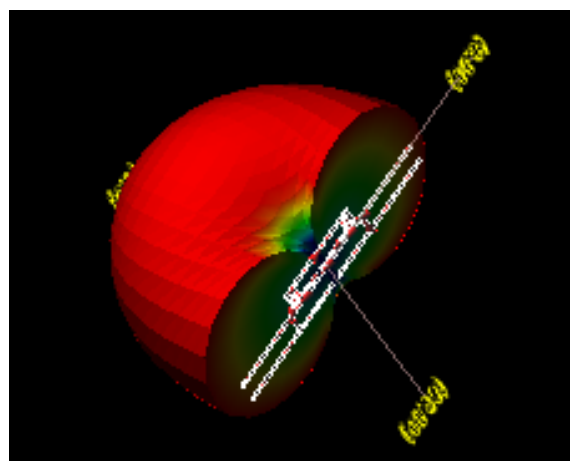

Fig.5 3D Radiation Pattern

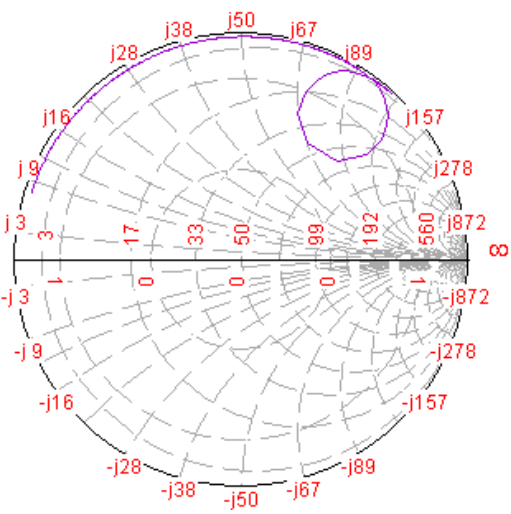

Fig.6 Smith Chart

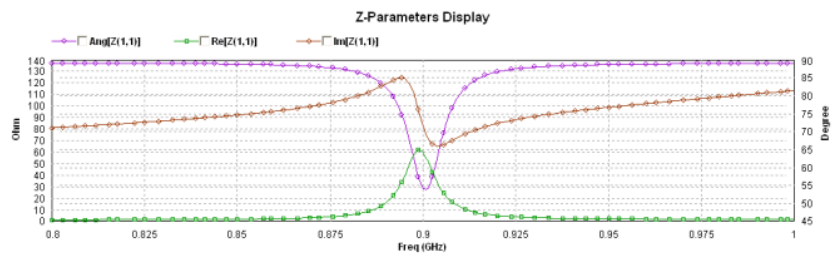

Fig.7 Input Impedance Characteristics

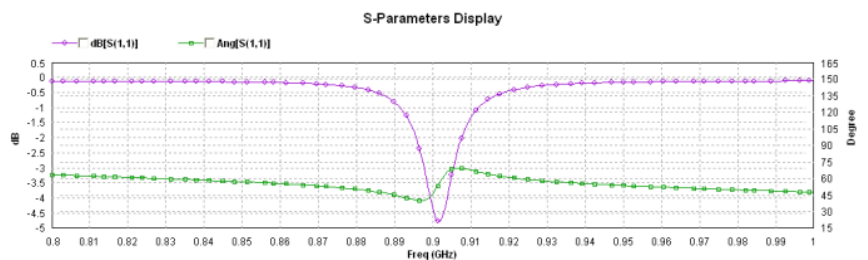

Fig. 8 S-Parameters

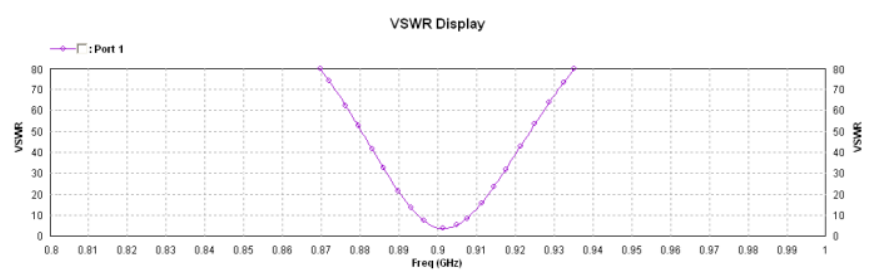

Fig. 9 VSWR

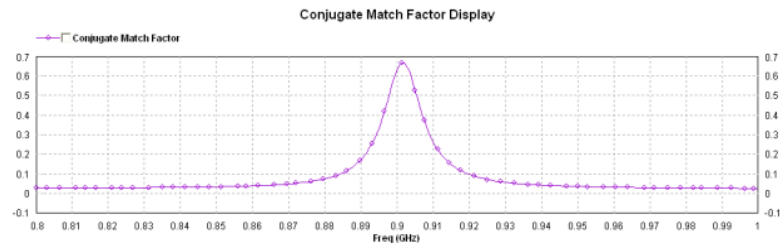

Fig. 10 Conjugate Matching Factor

\section{CONCLUSION}

The proposed novel indirect coupling method for the RFID tag antenna design, which provides a relatively straightforward tuning of the input impedance of the RFID tag antenna. Radio frequency identification is a rapidly developing technology, and it will find more and more applications. Besides RFID chip, RFID tag antenna also plays 
an important role in RFID tag. In general, the impedance of antenna to be a real number, but in RFID tag the required impedance to be a conjugate to chip's complex impedance. During the design process, it is important to monitor the antenna impedance as close as possible to conjugate match with chip over the operating frequency.

\section{ACKNOWLEDGMENTS}

We are heartily thankful to our supervisors, friends and family members, whose encouragement, guidance and support from the initial to the final level enabled us to develop an understanding of the subject. Lastly, we offer our regards and blessings to all of those who supported us in any respect during the completion of this paper.

\section{REFERENCES}

[1] Wen-Shan Chen*, Jhih-Ciang Chen, Bau-Yi Lee, Department of Electronic Engineering, Southern Taiwan UniversityYung-Kang city, Tainan County 710, Taiwan, R.O.C. "A Compact UHF RFID Tag Antenna Design for Metallic Objects", IEEE 2010

[2] Y. Choi, U. Kim, J. Kim and J. Choi, "Design of modified folded dipole antenna for UHF RFID tag", Electronics Letters, 9th April 2009, Vol. 45 No. 8.

[3] Youbok Lee, Ph.D. Microchip Technology Inc. "Antenna Circuit Design for RFID Applications" 2003, Microchip Technology Inc.

[4] C.-H. Loo, K. Elmahgoub, F. Yang, A. Elsherbeni, D. Kajfez A. Kishk and T. Elsherbeni, Department of Electrical Engineering, University of Mississippi University, MS 38677, USA, "Chip impedance matching for UHF RFID tag antenna design", Progress In Electromagnetics Research, PIER 81, 359-370, 2008

[5] Pavel V. Nikitin, Senior Member, IEEE, K. V. Seshagiri Rao, Senior Member, IEEE, Rene Martinez, Member, IEEE, and Sander F. Lam, "Sensitivity and Impedance Measurements of UHF RFID Chips", IEEE Transactions on Microwave theory and techniques, Vol. 57, No. 5, May 2009.

[6] Sonnet Application Note: SAN-206A,"Using Electromagnetic Analysis for RFID Antenna Design", Feb 2006, Sonnet Software, Inc.

[7] Johan Sidén and Hans-Erik Nilsson Mid Sweden University / Sensible Solutions Sweden AB Sweden, "RFID Antennas - Possibilities and Limitations".

[8] K. Finkenzeller, "RFID Handbook", ISBN 0-470-844027, John Wiley \& Sons 2003.

[9] C.A. Balanis, "Antenna Theory: Analysis and Design, 2nd ed.", ISBN 0-471-59268-4, John Wiley \& Sons 1997.

[10] Albert Lozano-Nieto, “ RFID Design Fundamentals and Applications", International Standard Book Number: 978-1-4200-9125-0 (Hardback), (C) 2011 by Taylor and
Francis Group, LLC CRC Press is an imprint of Taylor \& Francis Group an Informa business.

[11] Dominique Paret Consultant - Senior Technical Expert, DP-Consulting, "RFID at ultra and super high frequencies Theory and application", John Wiley \& Sons 2009.

[12] IE3D Benchmark Simulation Examples, Zeland Software, Inc.\& bay technology.

[13] White paper on common RFID implementation issues, September 2007, Alien Technology, Morgan Hill, CA 95037.

\section{AUTHORS BIOGRAPHY}

J. Gandhimohan received B.Tech degree in Electronics and Communication Engineering from Pondicherry University, India, in 2000. He received the the Diploma in Electronics and Communication Engineering from Tamilnadu Technical Board, Chennai, India in 1995. He started his teaching career at Government Polytechnic Colleges, Puducherry in the year 2001. He is generally interested in Communication Systems. His current work includes an antenna design for various wireless applications and specially for RFID's. He presented a paper on "UHF RFID tag dipole antenna using indirect coupling" in International conference for Microwave, Antenna and Remote Sensing at Rajasthan, India in 2011.His past work includes design of SSB Transceiver for the range of $50 \mathrm{~km}$ at diploma level and design of Wireless in Local Loop for the PSTN line at Graduate level. He guided more than 30 projects for the diploma students in his teaching experience. Currently he is doing M.Tech (Electronics) at Pondicherry University, India, under the Quality Improvement scheme.

T. Shanmuganantham was born May 12, 1974. He received B.E. degree in Electronics and Communication Engineering from University of Madras in 1996, M.E. degree in Communication Systems from Madurai Kamaraj University in 2000 and Ph.D. degree (Gold Medal) in the area of Antennas from National Institute of Technology, Tiruchirappalli, India under the guidance of Dr.S.Raghavan, Professor of ECE. He has 15 years of teaching experience in various reputed Engineering colleges such as SSN College of Engineering, Chennai, National Institute of Technology and Science, Chennai, PKIET, Karaikal. He has been with the Department of Electronics Engineering, School of Engineering \& Technology, Pondicherry University, Pondicherry as an Assistant Professor since 2010. His research interest includes Antennas, Microwave \& Millimeter-wave Engineering, Microwave Integrated Circuits, MEMS/NEMS, Metamaterials, Computational Electromagnetics, EMI/EMC, RF MEMS, RFIC Design, Optical Communication, Soft Computing for RF \& Microwave Engineering, Terahertz Technology. He has published 40 research papers in various national and International level Journals and Conferences. He is a member in IEEE, Life Member in IETE, Institution of Engineers, CSI, Society of EMC, ISTE, ISSS, OSI, ISI and ILA. 